\title{
Time analysis of the light pulses on gaseous active scintillators using GEMs with $\mathrm{He} / \mathrm{CF}_{4}$
}

\author{
L.M.S. Margato ${ }^{a} *$ F.A.F. Fraga ${ }^{a}$, S.T.G. Fetal ${ }^{a}$, M.M.F.R. Fraga ${ }^{a}$, \\ R. Ferreira Marques ${ }^{\mathrm{a}}$, A.J.P.L Policarpo ${ }^{\mathrm{a}}$, B. Guerard ${ }^{\mathrm{b}}$, G. Manzin ${ }^{\mathrm{b}}$ \\ ${ }^{\text {a }}$ LIP, Coimbra and Departamento de Física da Universidade de Coimbra, Coimbra 3004-516, Portugal \\ ${ }^{\mathrm{b}}$ Institute Laue Langevin, BP 156X, Grenoble Cedex F-38042, France
}

\begin{abstract}
The time structure of light pulses from GEM detectors operated in scintillation mode with $\mathrm{He} / \mathrm{CF}_{4}$ mixtures, previously used with integrating readout devices for neutron detection, has been investigated. A system using photomultipliers (PMs) and charge preamplifiers coupled to a fast digitizer has been used to measure simultaneously both the charge and light signals of the avalanches and the dependence of these signals on the operating conditions of the GEM, such as gain, transfer yield and collection field. The present results show that detectors relying on photon counting techniques, using of position sensitive PMs or arrays of avalanche photodiodes, are most promising as fast position sensitive neutron detectors.
\end{abstract}

(C) 2003 Elsevier Science B.V. All rights reserved.

PACS: 29.40.Cs; 29.40.Gx; 29.40.Mc

Keywords: GEM detectors; CCD; Microstructures; Scintillation

\section{Introduction}

The next generation of spallation neutron sources is underway, e.g. the ESS, the SNS and the Arena facilities. Their high neutron fluxes coupled to the need of a large area $\left(\sim 6 \mathrm{~m}^{2}\right)$ position sensitive neutron detection require new principles of neutron detection. Gaseous neutron detectors, usually associated with low cost high efficiency and high gamma rejection, are suited for developing large area detectors.

*Corresponding author. Tel.: + 351-39-833-465; fax: + 35139-822-358.

E-mail address: margato@lipc.fis.uc.pt (L.M.S. Margato).
Our recent studies have shown that avalanche scintillation in $\mathrm{He}-\mathrm{CF}_{4}$ is sufficiently high to allow the development of detectors with CCD readout [1] and we could obtain images of the proton and triton tracks in ${ }^{3} \mathrm{He}-\mathrm{CF}_{4}$ mixtures. It was also shown that the light intensity along the track could be used to determine exactly the interaction point and that the scintillation light produced by the neutron interaction in an active gas scintillator is over $10^{3}$ higher than the best existing passive scintillators [2].

In many experiments neutron detectors must be capable of performing high background rejection, mainly gammas, which is impossible to accomplish with integrating readouts. However, this task could be done with a photon counting readout making use of position sensitive photomultipliers 
(PMs) or arrays of avalanche photodiodes (APDs). To allow operation at high-count rates the light signals from the GEM based active scintillators should be fast and have no slow components.

A system using PMs (Philips 56TUVP-type B) and charge preamplifiers coupled to a fast digitizer (Tektronix TDS 7104) has been assembled to study simultaneously the time structure of light and charge signals of a double GEM detector.

\section{The experimental set-up}

The diagram of the system used in this work is shown in Fig. 1. The stainless steel chamber is able to support a pressure up to $10 \mathrm{bar}$, and is fitted with a $0.5 \mathrm{~mm}$ carbon fiber window on the radiation entrance side and a $12.5 \mathrm{~mm}$ thick quartz window with an effective diameter of $5 \mathrm{~cm}$, for the output of the scintillation light.

The feedthroughs were glass insulated. The detector was operated in a closed system mode and the gases used, all high purity research grade, were supplied to the chamber through stainless steel pipes without further purification.

A drift grid placed before the first GEM, defined a $5 \mathrm{~mm}$ conversion region. The GEMs were supported by ceramic frames defining a $2 \mathrm{~mm}$ zone between the first and the second GEM, the transfer



Fig. 1. Diagram of the experimental system. The avalanche scintillation light was detected with a photomultiplier Philips 56TUVP-type B. A Tektronix TDS 7104 oscilloscope $(1 \mathrm{GHz}$, $10 \mathrm{GS} / \mathrm{s}$ ) was used to digitize both light and charge signals. region, and an optically transparent collecting grid was placed $2 \mathrm{~mm}$ far away from the second GEM, defining the induction gap. GEMs with an area of $5 \times 5 \mathrm{~cm}^{2}$ having 80 and $60 \mu \mathrm{m}$ metal diameter holes with, respectively, 70 and $50 \mu \mathrm{m}$ bi-conical kapton holes were used. The kapton thickness was $50 \mu \mathrm{m}$ and pitch was $140 \mu \mathrm{m}$ for all GEMs. A PM (Philips 56TUVP-type B-extended S20) has been coupled to the quartz window of the detector for the readout of the scintillation light.

The primary electron clouds produced by X-ray photons in the conversion region are focused in the channels of the first GEM, where the first charge multiplication process occur. Using an appropriate electric field, $E_{\mathrm{t}}$, a fraction of these electrons is transferred into the channels of the second GEM. For the $\mathrm{He}+40 \% \mathrm{CF}_{4}$ mixture at 1 bar, the optimum transfer field is $E_{\mathrm{t}} \sim 2 \mathrm{kV} / \mathrm{cm}$, transferring about $25 \%$ of the charge produced in the first GEM. The electrons injected in the second GEM are multiplied again and the scintillation emitted by the electron avalanches is detected by the PM.

Depending on the magnitude of the induction field, a fraction of these electrons, produced in the second GEM, drift towards the induction electrode (with a null induction field all the electrons would be collected at the outer electrode of the second GEM), inducing a signal on it. If, as usually done, the induction gap is operated in the ionization mode, the rise time of the signal is determined by the drift of electrons across this gap and depends on the value of the reduced field, gaseous mixture and gap dimensions.

Both signals from the induction grid (denominated charge signals) and from the PMT (denominated light signals) have been amplified by charge preamplifiers (PA) and were further digitized with a Tektronix TDS7104 scope (see Fig. 1).

The time response of the preamplifiers has been determined with a fast-calibrated pulse, the rise times are $14 \mathrm{~ns}$ for the Camberra 2005 and $24 \mathrm{~ns}$ for the Camberra 2006.

\section{Energy resolution}

The poor energy resolution of $\mathrm{CF}_{4}$ based mixtures is due to the electron attachment, and a 
study carried out with proportional counters shows that the measured energy resolution is not better than $18 \%$ at $5.9 \mathrm{keV} \mathrm{[3].}$

The energy resolution of the double GEM system with a ${ }^{55} \mathrm{Fe}$ source has been measured for both charge and light signals. Fig. 2 shows that, using a $5.9 \mathrm{keV} \mathrm{X}$-ray source, an energy resolution of $20 \%$ can be obtained for both charge and light signals.

Fig. 3 shows the energy resolution variation as a function of the double GEM gain. The small improvement in energy resolution with higher gain when using the light signals is due to the better statistics of the higher number of detected photons. As expected, once the number of detected photons is sufficiently high the energy resolution of the light signals follows the behavior of the charge mode.

\section{Study of the rise time}

It is known that $\mathrm{CF}_{4}$ is a fast scintillator and that the measured radiative lifetimes at a pressure of $10 \mathrm{~m}$ Torr are in the ns range [4].

The typical signal shape of the light signals measured at the output of the preamplifier is shown in Fig. 4a. Fig. $4 \mathrm{~b}$ shows the distribution of the rise times. For typical induction fields and gap dimensions the light signals are faster than charge signals, as confirmed in Fig. 5.

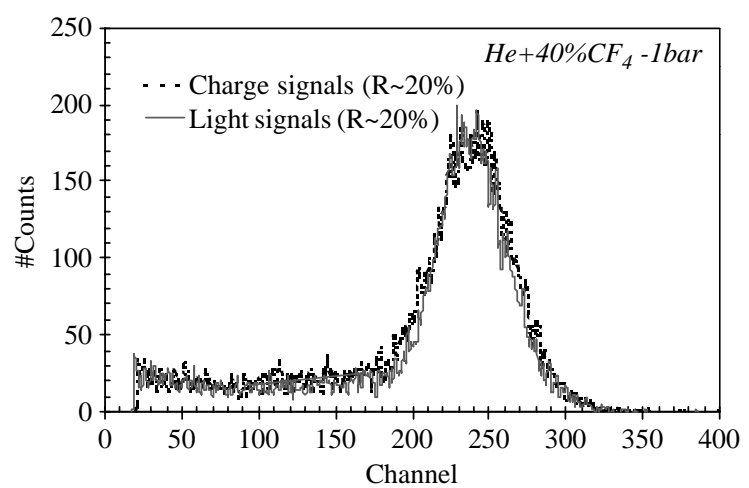

Fig. 2. Charge and light pulse-height spectra obtained using a $5.9 \mathrm{keV}{ }^{55} \mathrm{Fe}$ X-ray source: $E_{\mathrm{d}}=0.5 \mathrm{kV} / \mathrm{cm}, E_{\mathrm{t}}=E_{\mathrm{i}}=2 \mathrm{kV} / \mathrm{cm}$, $V$ gem $_{1}=V$ gem $_{2}=460 \mathrm{~V}$; double GEM gain $\sim 9 \times 10^{4}$.

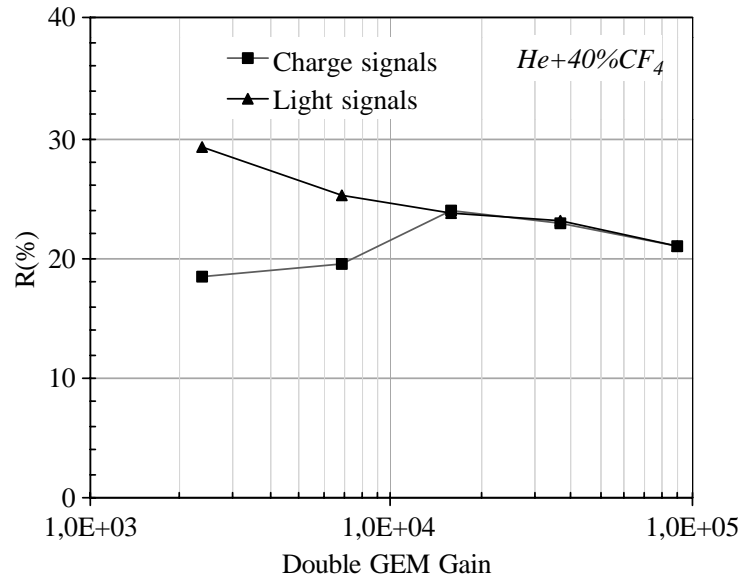

Fig. 3. Charge and light energy resolution versus double GEM gain for $5.9 \mathrm{keV}{ }^{55} \mathrm{Fe}$ X-ray source: $E_{\mathrm{d}}=0.5 \mathrm{kV} / \mathrm{cm}$, $E_{\mathrm{t}}=E_{\mathrm{i}}=2 \mathrm{kV} / \mathrm{cm}$.

(a)
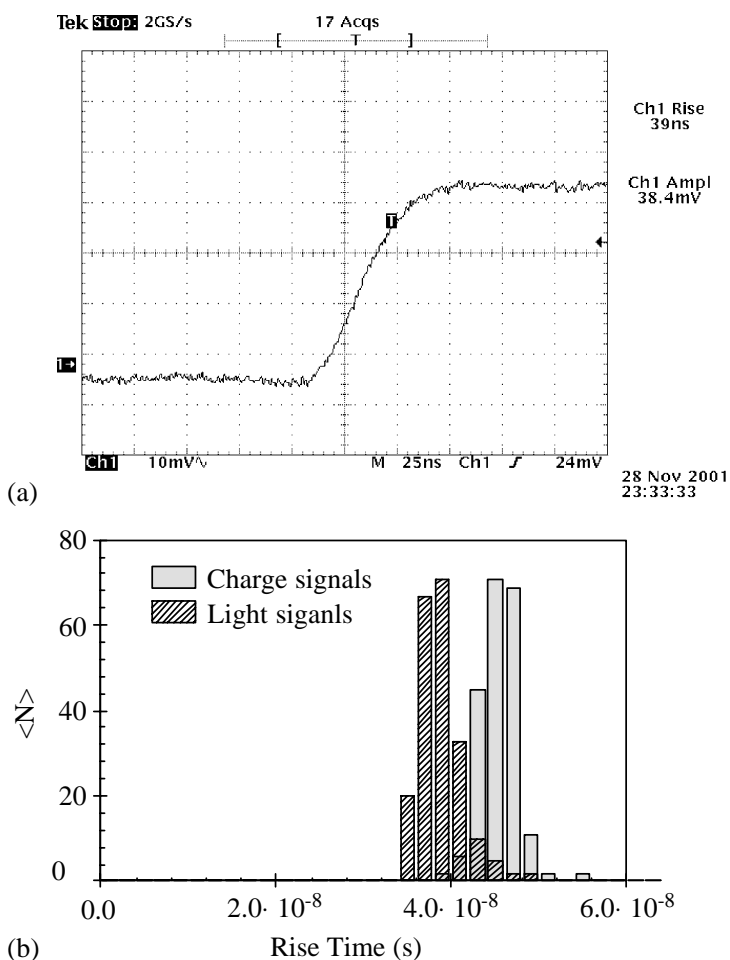

Fig. 4. Experimental conditions: $E_{\mathrm{d}}=0.5 \mathrm{kV} / \mathrm{cm}, E_{\mathrm{t}}=\mathrm{kV} / \mathrm{cm}$, $E_{\mathrm{i}}=4 \mathrm{kV} / \mathrm{cm}, V$ gem $_{1}=390 \mathrm{~V}, V$ gem $_{2}=380 \mathrm{~V}$. (a) Typical signal shape of light signals, (b) distribution of the rise time of the light and charge signals taken with a X-ray photon energy of $5.9 \mathrm{keV}$. 
The dependence of the rise time on the induction field and the double GEM gain was measured and is shown in Figs. 5 and 6, after deconvolution of the rise time of the preamplifiers. The dependence of the rise time of charge signals on the intensity of the induction field is consistent with the transport parameters given by Kopp et al. [5].

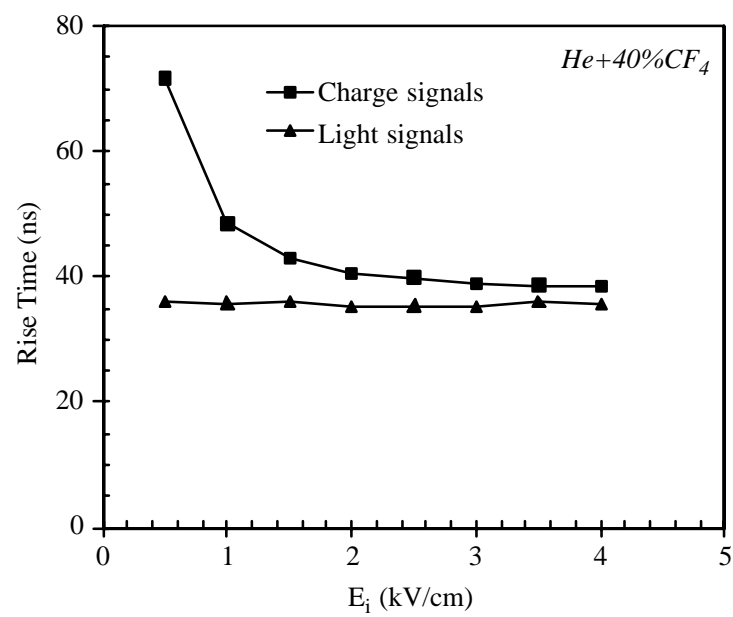

Fig. 5. Average rise time of the light and charge signals versus induction field, $E_{\mathrm{i}}$, between second GEM and induction grid. A $5.9 \mathrm{keV}$ X-ray ${ }^{55} \mathrm{Fe}$ source was used. $E_{\mathrm{d}}=0.5 \mathrm{kV} / \mathrm{cm}$, $E_{\mathrm{t}}=E_{\mathrm{i}}=2 \mathrm{kV} / \mathrm{cm}, V$ gem $_{1}=390 \mathrm{~V}$ and $V$ gem $_{2}=380 \mathrm{~V}$; double GEM gain $\sim 3.1 \times 10^{3}$.

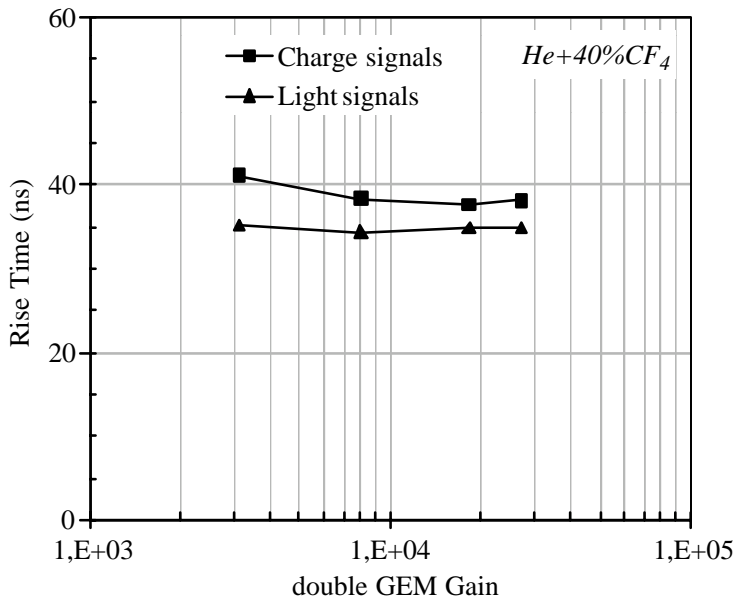

Fig. 6. Average rise time of the light and charge signals as a function of the double GEM effective gain. A $5.9 \mathrm{keV}$ X-ray ${ }^{55} \mathrm{Fe}$ source was used. $E_{\mathrm{d}}=0.5 \mathrm{kV} / \mathrm{cm}, E_{\mathrm{t}}=E_{\mathrm{i}}=2 \mathrm{kV} / \mathrm{cm}$ and $V$ gem $_{1}=390 \mathrm{~V}$.
The results of Fig. 5 show that light signals have rise time of less than $36 \mathrm{~ns}$ and that, as expected, do not depend on the collection field, unlike the charge signals.

From Fig. 6 it can be seen that the rise time of both types of signals does not depend strongly on the double GEM gain.

We also studied the dependency of the light signals rise time on the X-ray energy. An X-ray tube was used to generate a broad $\mathrm{X}$-ray spectrum with a maximum energy of $25 \mathrm{keV}$ and the trigger level of the digitizing oscilloscope has been used as an energy discriminator for this analysis.
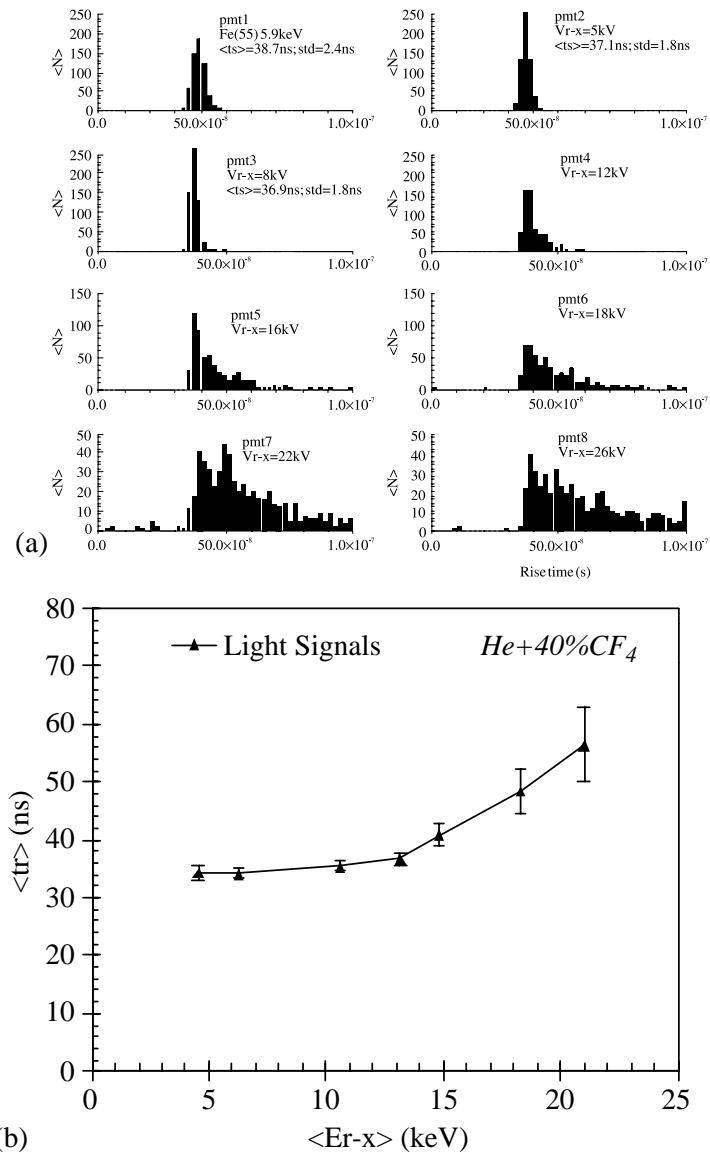

Fig. 7. Polarization condition: $E_{\mathrm{d}}=0.5 \mathrm{kV} / \mathrm{cm}, \quad E_{\mathrm{t}}=E_{\mathrm{i}}=$ $2 \mathrm{kV} / \mathrm{cm}, V$ gem $_{1}=390 \mathrm{~V}$ and $V$ gem $_{2}=380 \mathrm{~V}$; double GEM gain $\sim 3.1 \times 10^{3}$. (a) Distribution of the rise time of light signals for several X-ray generator high voltages, (b) average rise time of light signals versus X-ray energy. 
As expected, taking in consideration the dependence of the range of the primary electrons on the $\mathrm{X}$-ray energy [6], the rise time distributions get broader with X-ray energy. The longer rise times are associated with primary tracks having a larger component on the drift direction, as shown in Fig. 7a. This behavior is highlighted in Fig. 7b that shows the dependency of the mean rise time and associated broadening versus X-ray energy.

\section{Conclusions}

In this work it has been shown that, using a double GEM in a $\mathrm{He} / \mathrm{CF}_{4}$ mixture, the light signals have a rise time of less than $40 \mathrm{~ns}$, and are even more rapid than the charge signals. It was also shown that, in photon counting mode, time and energy resolution are compatible with applications that can take advantage of energy discrimination capabilities such as thermal neutron imaging.

GEMs operated in light mode can be used with other scintillation mixtures and readout by position sensitive PMs or APD arrays. These detectors could be used for improved 3D track localization purposes in applications such as neutron spectroscopy and even X-ray polarimetry.

\section{Acknowledgements}

This work has been supported by the contract CERN/FNU/43735/2001 with the Portuguese FCT.

L.M.S. Margato has been supported by the FCT (Fundação para a Ciência e Tecnologia) SFRH/ $\mathrm{BD} / 6786 / 2001$ research grant.

\section{References}

[1] F.A.F. Fraga, et al., Nucl. Instr. and Meth. A 471 (2001) 125.

[2] F.A.F. Fraga, L.M.S. Margato, S.T.G. Fetal, M.M.F.R. Fraga, R. Ferreira Marques, A.J.P.L. Policarpo, B. Guerard, A. Oed, G. Manzini, T. Van Vuure, Nucl. Instr. and Meth. A 478 (2002) 357.

[3] W.S. Anderson, J.C. Armitage, E. Dunn, J.G. Heinrich, C. Lu, K.T. McDonald, J. Weckel, Y. Zhu, Nucl. Instr. and Meth. A 323 (1992) 273.

[4] A. Pansky, A. Breskin, A. Buzulutskov, R. Chechik, V. Elkind, J. Va'vra, The scintillation of $\mathrm{CF}_{4}$ and its relevance to detection science, WIS-94/23/May-PH.

[5] M.K. Kopp, K.H. Valentine, L.G. Christophorou, J.G. Carter, Nucl. Instr. and Meth. A 201 (1982) 395.

[6] J. Fischer, V. Radeka, G.C. Smith, Nucl. Instr. and Meth. A 252 (1986) 239. 